\title{
Fabrication and Encapsulation of a Short-Period Wire Grid Polarizer with Improved Viewing Angle by the Angled-Evaporation Method
}

\author{
Young Jae Shin, Yi-Kuei Wu, Kyu-Tae Lee, Jong G. Ok, and L. Jay Guo*
}

\begin{abstract}
A wire grid polarizer (WCP) is fabricated by using a simplified approach with a combination of nanoimprint lithography (NIL) and angled metal deposition. The period of the imprinted polymer nanograting used in this study is $180 \mathrm{~nm}$. The WGP is formed by two consecutive angled aluminium evaporation processes, essentially halving the period for the aluminium (Al) nanograting. The fabricated WGP shows good optical properties for polarized light. More importantly, because of the slight reduction of the period of the nanograting as compared with previous results, the viewing angle, which is one of the most important characters in display equipment, is extended greatly. Encapsulation of the WGP using poly(methyl methacrylate) is conducted for practical application of the WGP. The thickness of the coating is controlled to be less than $1 \mu \mathrm{m}$, to prevent the degradation of the optical properties of the WGP.
\end{abstract}

spectral range and small size, and they can be integrated with other optical elements on the same chip. They can also be constructed as high-quality, integration-capable, thin-film-type polarizers..$^{[1-8]}$ WGPs can be applied to various fields such as microscopy, imaging systems, and polarized beam splitters. WGPs in the visible optical region were reported a decade ago by the MOXTEK group as beam splitters for projection displays..$^{[9]}$ WGPs may improve the brightness of LCDs because their function is not based on light absorption, and reflected light can be recycled. However, for direct-view liquid crystal displays, a WGP can only be used as a bottom polarizer but cannot replace

\section{Introduction}

The liquid crystal display (LCD) is currently the prevailing display technology. An LCD requires polarized illumination to function. Therefore, polarizers are a very important part of an LCD and are also essential components in many optical systems and networks. The optical performance of conventional polarizers based on dichroic absorption cannot meet the requirements of some new optoelectronic systems currently under development. A wire grid polarizer (WGP) is one of the most attractive alternatives. The WGP is made of parallel metallic lines on a transparent substrate and is used to transmit transverse magnetic (TM) polarized light (E-field perpendicular to the grating) and reflect transverse electric (TE) polarized light (E-field parallel to the grating direction). WGPs offer a large

\footnotetext{
Y. J. Shin, Prof. L. J. Guo

Macromolecular Sciences and Engineering

University of Michigan

Ann Arbor, Michigan, 48109, USA

E-mail: guo@umich.edu

Dr. Y.-K. Wu, K.-T. Lee, Prof. L. J. Guo

Department of Electrical Engineering and Computer Science

University of Michigan

Ann Arbor, Michigan, 48109, USA

J. G. Ok, Prof. L. J. Guo

Department of Mechanical Engineering

University of Michigan

Ann Arbor, Michigan, 48109, USA
}

DOI: 10.1002/adom.201300276 the top dichroic polarizers, because it would reflect ambient light. ${ }^{[10]}$ One potential solution was developed by adding an absorptive interference layer on top of the $\mathrm{Al}$ grating to reduce light reflection. ${ }^{[11]}$ Still much investigation in both design and fabrication are needed to push the WGP to practical application. Additionally, displays for mobile devices have progressed toward increasingly thinner, lighter, and more flexible devices. Future flexible displays could benefit from the WGPs made on a polymer substrate.

TM transmission and TE suppression are the most important features of WGPs, and hence the extinction ratio, which is defined as the ratio of TM transmission to the TE transmission, is also an important factor. The optical properties of a WGP are determined by the grating material, the period and line width of the grating, and the grating height. ${ }^{[12]}$ In the last two decades, various research results on the fabrication of WGPs using nanoimprint lithography (NIL) have been reported..$^{[1-8]}$ For example, a bilayer metal WGP was presented by Ekinci et al. ${ }^{[13]}$ The fabrication of a flexible nano-wired polarizer by contacttransferred and mask-embedded lithography using a polyurethane acrylate mold was described by Hsu et al. ${ }^{[14]}$ NIL has been used in recent years due to its potential for high resolution and high throughput nanoscale patterning to produce the fine pitch required for a WGP. ${ }^{[15-20]}$ We also reported previously the fabrication of bilayer metal WGPs on flexible plastic substrates by roll-to-roll UV nanoimprint lithography and the metal evaporation technique, ${ }^{[18]}$ and a WGP embedded in gas-permeable membrane for contact lens application. ${ }^{[12]}$ Particularly, the high throughput UV-NIL process has the potential to enable large area metal WGP fabrication. ${ }^{[21,22]}$ 
WGPs require fabrication of subwavelength metal gratings, which presents a significant challenge for patterning and etching of dense structures, particularly for use in visiblewavelength applications. It becomes even more challenging to fabricate these structures on a flexible substrate. It is known that the WGP should have a period shorter than $150 \mathrm{~nm}$ in order to provide adequate bandwidth to cover the visible light range. In our previous publication, ${ }^{[23]}$ a flexible WGP was fabricated on a polyethylene terephthalate (PET) film with an imprinted $220 \mathrm{~nm}$-period high-aspect-ratio polymer grating with double-angled aluminium deposition to cover the sidewalls of the imprinted polymer grating. Isotropic reactive ion etching (RIE) was then used to remove selectively the aluminium deposited on the top of the grating, thereby forming metal wire grids with spacing much closer than lithographically defined gratings. Even though the imprinted grating had a relatively large period of $220 \mathrm{~nm}$, the obtained WGP showed good performance in the wide visible band of the spectrum due to a much narrower spacing between the aluminium lines. As a result, good optical performance was obtained in the visible band of the spectrum. This approach significantly relaxes the stringent requirement for the fine line patterning process used in traditional methods, and is particularly desirable for future manufacturing of flexible WGPs using the roll-to-roll nanoimprint process. For practical application, encapsulation of the WGP is highly desirable to improve the mechanical integrity of the high aspect ratio metallic nanogratings and makes it easier to integrate with other optical components. However, we found that encapsulated WGP can exhibit color if not properly designed. Moreover the polarizer performance degrades when light is incident from a higher angle. This study shows that these issues can be addressed by reducing the period of the grating. In particular we fabricated the WGP using a grating mold with a $180 \mathrm{~nm}$ period to prove the principle.

\section{Results and Discussion}

\subsection{Simulation of Angle Dependency}

The WGP which was reported in our previous paper ${ }^{[23]}$ showed a large angle dependency and had a narrow viewing angle. Consequently, the TM transmission was reduced greatly even at a $30^{\circ}$ incident angle. A wide viewing angle is an important requirement for display devices such as an LCD. In order to improve the angle dependency of the WGP, we first tried simulations with a shorter period WGP. A nanoimprinted pattern with a $180 \mathrm{~nm}$ period, $70 \mathrm{~nm}$ linewidth, and $200 \mathrm{~nm}$ height was used as a model system, with $20 \mathrm{~nm}$ aluminium layer deposited along both walls of the patterned grating lines. Therefore the final WGP had a structure with periodic $20 \mathrm{~nm}$ wide aluminium gratings with $70 \mathrm{~nm}$ spacing.

Figure 1(a) and (b) show the simulation results of the angle dependency for the WGPs made from the polymer gratings with initial periods of 220 and $180 \mathrm{~nm}$, respectively. The simulation was conducted using the refractive index data of the base materials. The refractive index was measured by an ellipsometer (AutoEL), and the coating thickness was also measured. In Figures 1(a) and (b), the red-colored region indicates a high transmission of TM light, and the purple indicates low transmission of TM light. The overall angle dependency of the WGP with a $180 \mathrm{~nm}$ period was obviously improved than that of the WGP with a $220 \mathrm{~nm}$ period at visible wavelengths. For example, at $500 \mathrm{~nm}$, the transmission of the TM light improved greatly for the WGP with a $180 \mathrm{~nm}$ period at incident angles greater than $30^{\circ}$. In Figures 1 (c) and (d) the transmission levels of TE light are both below 0.008 and showed a similar pattern. Therefore the extinction ratio of the WGP also improves at a large viewing angle for shorter-period gratings. An explanation for this effect can be offered if we treat the wire grid layer as an effective medium. According to the paper, ${ }^{[24]}$ a sub-wavelength grating slab to a TM wave (grating period $d$, slit width of $a$ and slit depth of $h$ ) is almost equivalent to a dielectric plate with refractive index of $d / a$ and plate thickness of $h /(d / a)$. Based on this analogy, with decreasing $d / a$, the angledependence of transmission is weakened. Asymptotically, with $d / a$ approaching 1 , the slab is nothing but air and the angledependence should vanish completely. Therefore the reduction of spacing between the $\mathrm{Al}$ metal lines has a stronger effect than the reduction of the grating period in determining the angle dependence of the polarizer.

\subsection{Fabrication of the WGP}

An effective method for fabricating WGPs with shorter periods is to use double-angled aluminium depositions onto both sides of the nanoimprinted polymer grating walls. ${ }^{[23]}$ The aluminium at the upper part of the pattern can be removed by RIE. This process is shown in Figure 2. The final structure of the WGP has aluminium coatings on both sides of the nanoimprinted grating walls. A nanoimprinted pattern with a $180 \mathrm{~nm}$ period, $70 \mathrm{~nm}$ linewidth, and $200 \mathrm{~nm}$ height was prepared using NIL in this study to ensure adequate allowance for the aluminium coatings. Therefore, the final structure of the WGP after completing the entire process had a periodic $70 \mathrm{~nm}$ linewidth, $20 \mathrm{~nm}$ aluminium grating, $70 \mathrm{~nm}$ interval, and $20 \mathrm{~nm}$ aluminium grating, as shown in Figure 2. The overall fabrication process of the WGP is illustrated in Figure 2.

To imprint the polymer grating, we used silsesquioxane (SSQ) resist materials that were reported previously. ${ }^{[25,26]}$ Briefly, poly(phenyl-co-3-gylcidoxypropyl-co-perfluorooctyl) silsesquioxane (epoxy-SSQ) was synthesized from phenyltrimethoxysilane (PTMS), (tridecafluoro-1-octyl)triethoxysilane (FTES), and (3-glycidyloxypropyl)trimethoxysilane (GTMS). The PTMS component helps to increase the hardness of the synthesized SSQ polymer, and FTES helps to improve the releasing property of the imprinted SSQ patterns from the mold. GTMS contains an epoxy functional group which can polymerize during NIL via cationic polymerization. The epoxy functional group shows good mechanical properties after the completion of curing and simultaneously shows good adhesion. ${ }^{[23,25,26]}$ This property plays an important role in the NIL process to increase the adhesion of the imprinted material to the substrate, particularly for a dense, narrow linewidth and the high aspect ratio features required for a WGP. Better mold 


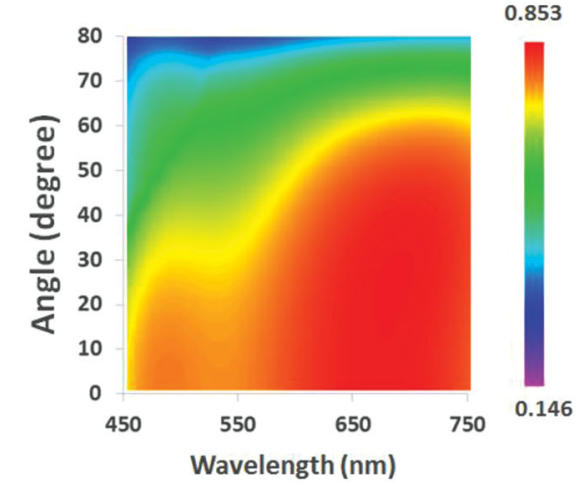

(a)

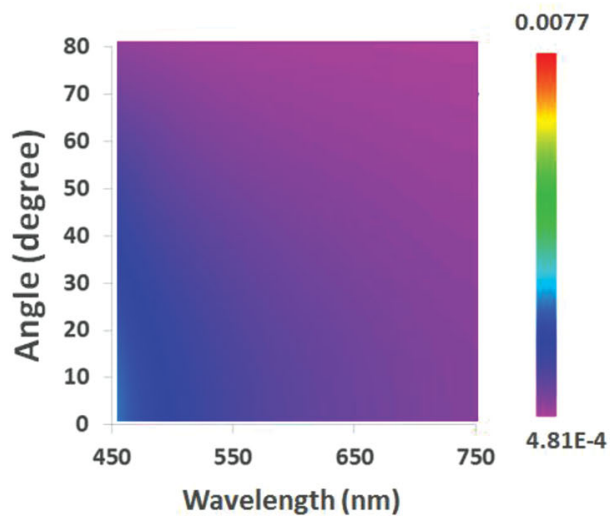

(c)

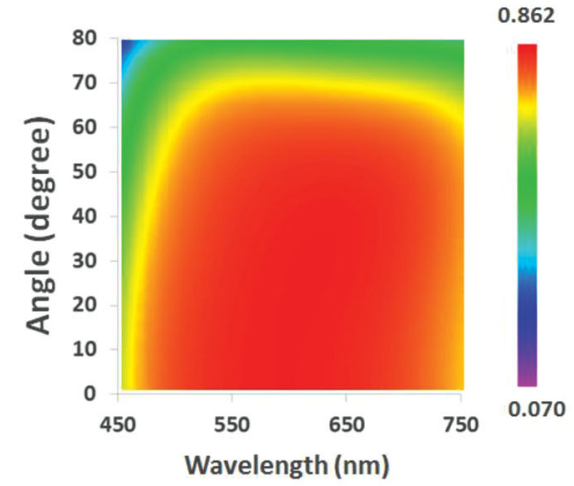

(b)

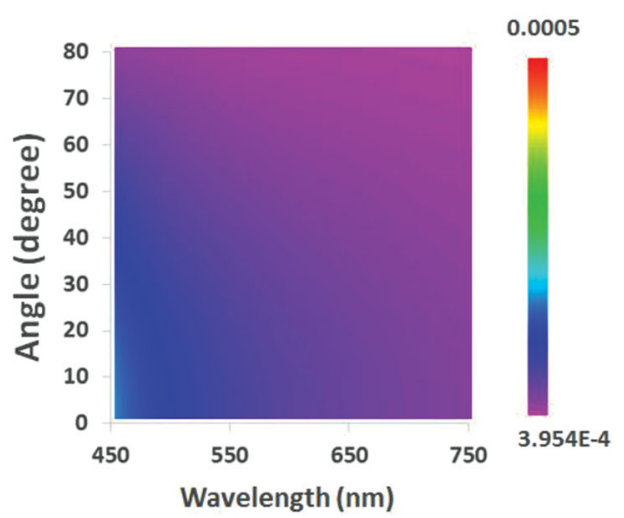

(d)

Figure 1. Simulation results showing the angle dependency for WGPs of $220 \mathrm{~nm}$-period polymer grating [TM transmission (a) and TE transmission (c)], and by a $180 \mathrm{~nm}$-period polymer grating [TM transmission (b) and TE transmission (d)]. The height of the nanograting is $200 \mathrm{~nm}$, and the thickness of the aluminium coating on the grating sidewall is $20 \mathrm{~nm}$.

releasing is also very important to ensure defect-free patterning over a large area. Figure $3(\mathrm{a})$ is a scanning electron microscope (SEM) image of the formed SSQ nanograting pattern showing a well defined and uniform high aspect ratio nanopattern with a 70-nm linewidth and $180 \mathrm{~nm}$ period. The SEM image of the fabricated WGP after double side angled aluminium deposition at 40 degrees and after RIE are shown in Figures 3(b) and (c),

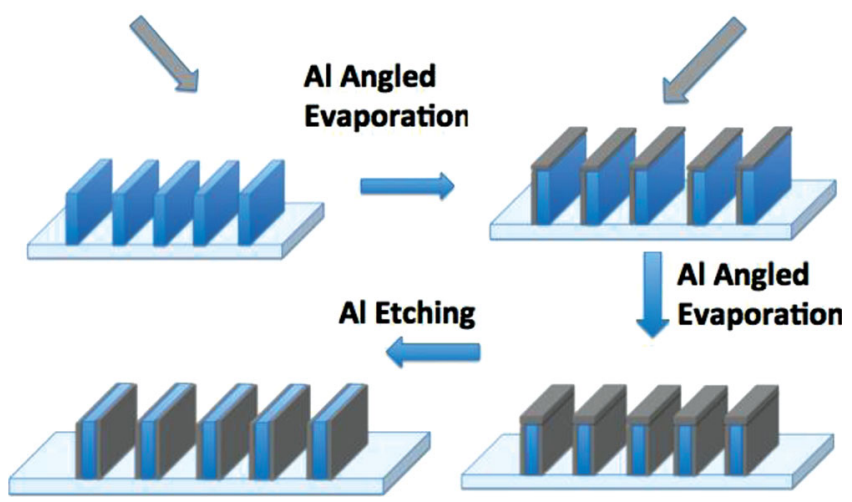

Figure 2. Double-angled evaporation of aluminium onto a nanopattern and the reactive ion etching process. respectively, where the aluminium layers on both side walls of the nanopatterns can be clearly seen.

\subsection{Optical Properties of the WGP}

The optical properties of the obtained WGP were characterized by measuring the transmittance of both the TM and the TE of polarized light. The results of the measurement of TM and TE transmission for the fabricated WGPs are listed in Table 1, in the visible range for the WGPs shown in Figure 3. In Figure 4, $80-90 \%$ of the TM polarized light was transmitted in the wide visible wavelength range of $450-700 \mathrm{~nm}$. Only $0.5-2 \%$ of the TE polarized light was transmitted in the same wavelength range. Optimization of the structure and fabrication process will further improve the WGP performance.

The angle dependencies of the 220 and $180 \mathrm{~nm}$ period WGPs were measured and shown in Figure 5. In the case of the WGP fabricated from the imprinted $220 \mathrm{~nm}$ period grating, the TM transmission amplitude drops severely with increasing incident angle for shorter wavelengths. For example, the transmission of TM light was reduced abruptly for $450 \mathrm{~nm}$ wavelength light as the angle was increased to $60^{\circ}$. The reduction was less servere at $550 \mathrm{~nm}$ as the angle was increased to $60^{\circ}$. Further, no 


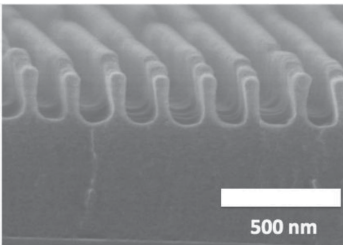

(a)

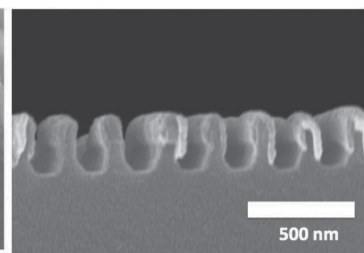

(b)

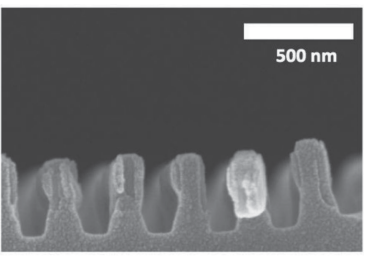

(c)

Figure 3. (a) SEM image of an SSQ nanopattern on a flexible PET film, and the cross section of a nanopattern (b) after aluminium deposition and (c) after RIE etching of the Al layer on top of the Al grating.

Table 1. Measured optical transmission [\%] of TM/TE polarized light through nanopatterned epoxy-SSQ/aluminium WGPs.

\begin{tabular}{lcccc}
\hline & $450 \mathrm{~nm}$ & $550 \mathrm{~nm}$ & $650 \mathrm{~nm}$ & $750 \mathrm{~nm}$ \\
\hline $\mathrm{TM} / \mathrm{TE}$ & $79.4 / 1.03$ & $83.1 / 0.74$ & $88.4 / 0.67$ & $79.7 / 7.47$ \\
\hline
\end{tabular}

significant reduction was observed at $650 \mathrm{~nm}$. This is because the wavelength is much larger than the $\mathrm{Al}$ grating spacings. It implies that reducing the grating period should improve the angle performance for shorter wavelength range. Indeed, for the WGP fabricated from the $180 \mathrm{~nm}$ period grating, the transmission of TM light was not reduced at any visible wavelength as the angle was increased to $60^{\circ}$.

Finally, we would like to discuss the optical performance of the WGP after the fabricated Al nanogratings are embedded in a polymer layer for protection. Such encapsulation of the WGP is very important for practical use; however, the encapsulating material must have minimal effect on the optical property of the WGP. A simulation was conducted for a WGP structure with a $90 \mathrm{~nm}$ period, $20 \mathrm{~nm}$ wide Al grating and, $200 \mathrm{~nm}$ height after encapsulation by materials with refractive indexes of 1.0 (e.g. air), 1.5 (typical polymer), and 2.0 (high index material). The results are shown in Figure 6. The results showed

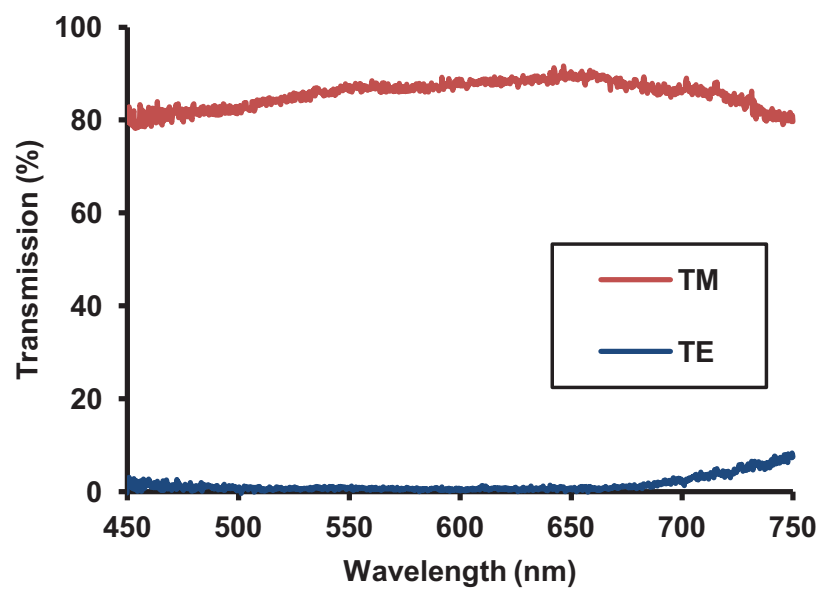

Figure 4. The measured $T E$ and $T M$ transmission of a nanopatterned epoxy-SSQ WGP with $20 \mathrm{~nm}$ aluminium deposition on both sidewalls (180 nm period, $70 \mathrm{~nm}$ linewidth, $200 \mathrm{~nm}$ grating height).

mission will be the subject of a subsequent paper. ${ }^{[27]}$

that when the refractive index of the encapsulating material is 2.0 , the TE transmission is not blocked, and therefore material of high refractive index cannot be used for encapsulation of a WGP. However, when the refractive index of the encapsulating material is 1.5 , the effect is much less, except for very short wavelength range. Therefore, we chose PMMA $(\mathrm{n}=1.49)$ as the encapsulating material for the WGP. More detailed studies on the physical mechanism of the transition from broadband polarizer to resonant trans-

Encapsulation of the WGP with PMMA was conducted by the dip-coating method. The coating thickness was controlled to be less than $1 \mu \mathrm{m}$. A simulation was conducted to estimate the effect of the coating thickness on TM and TE. The results are shown in the supporting information. The transmission of TE and TM light were then measured for the encapsulated WGP. The result is shown in Figure 7. Nearly all of the data plotted in Figure 7 are similar to the data plotted in Figure 3. This implies that the encapsulation of the WGP in PMMA does not affect the performance of the WGP.
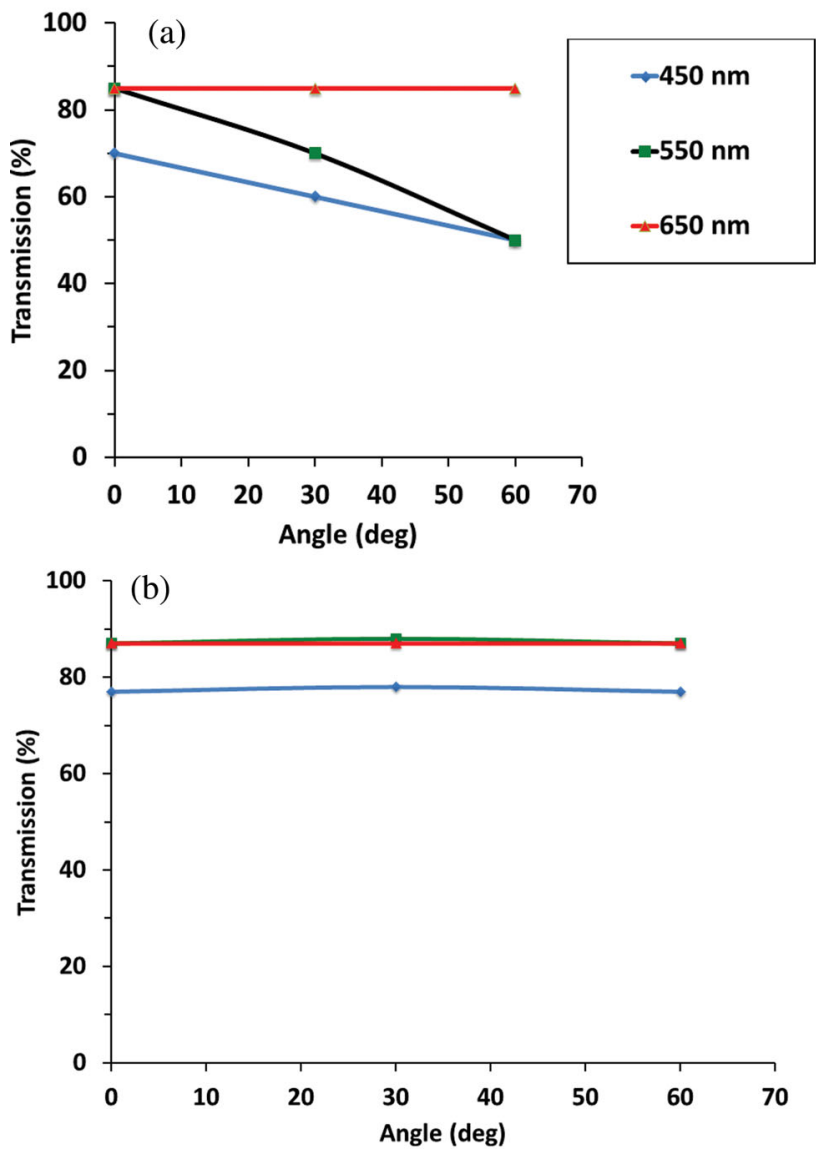

Figure 5. The transmission of TM light for (a) the 220-nm period WGP and (b) the 180-nm period WGP. 


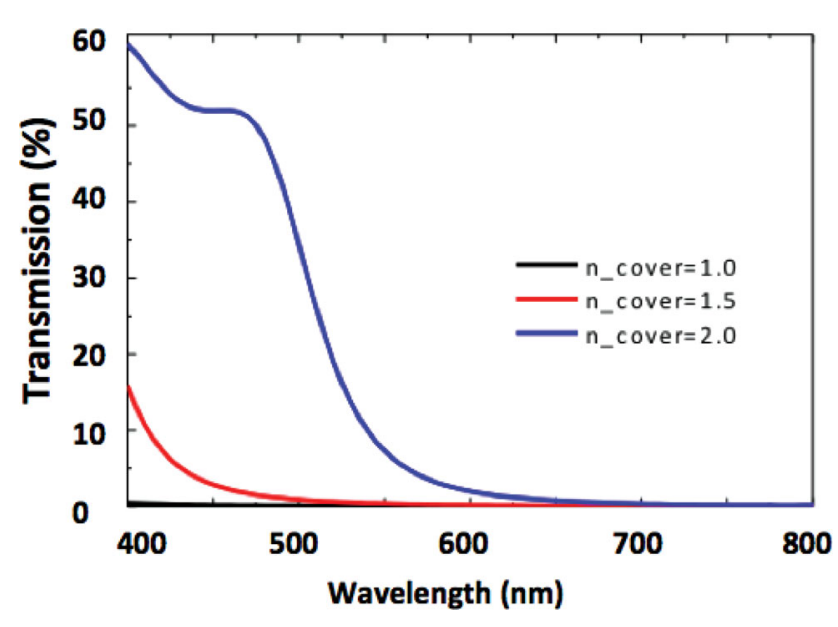

Figure 6. The simulated TE transmission of a WGP encapsulated with materials of refractive indices of 1.0, 1.5, and 2.0.

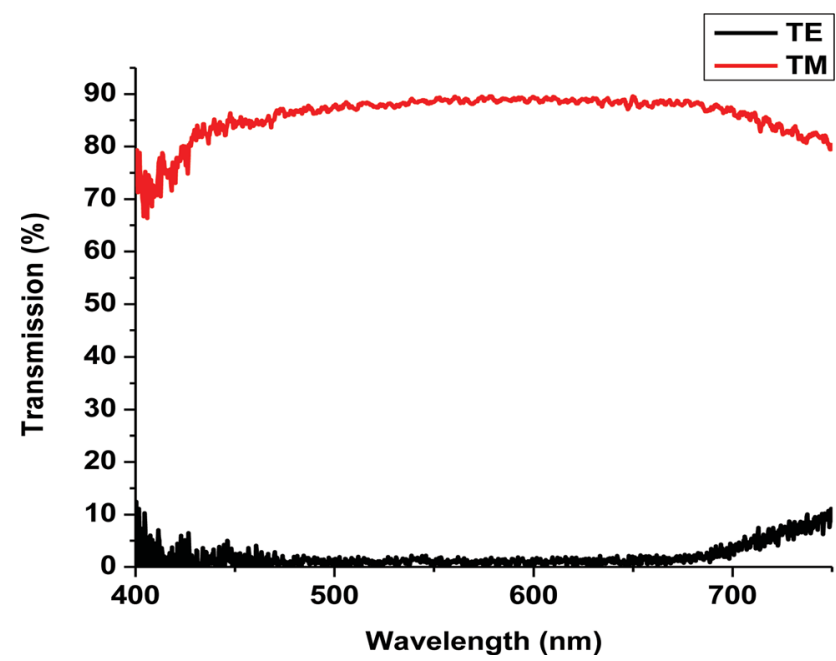

Figure 7. The TE and TM curves of a nanopatterned epoxy-SSQ WGP after PMMA encapsulation.

\section{Conclusion}

In this study, a WGP was fabricated by nanoimprint lithography, double-side angled evaporation of aluminium, and anisotropic RIE to remove the aluminium deposited on the top of the grating. The final structure of the $\mathrm{Al}$ grating has a $90 \mathrm{~nm}$ period, and about $20 \mathrm{~nm}$ aluminium linewidth. This WGP showed good performance in a wide range of visible wavelengths, which is not affected much at increased viewing angle. We also showed that the WGP can be encapsulated in PMMA for practical use.

\section{Experimental Section}

Materials and the Synthesis of Imprinting Material: Phenyltrimethoxysilane (PTMS), (3-glycidyloxypropyl)trimethoxysilane (GTMS), (tridecafluoro-1-octyl)triethoxysilane (FTES), $\mathrm{CsOH}$, and propylene glycol monomethyl ether acetate (PGMA) were purchased from Aldrich Chemical (St. Louis, MO, USA). Photoacid generator
(PAG) was purchased from Craig Adhesive and Coating Co. (Newark, NJ, USA) under the compound name of UV9390C. It contained $\sim 50 \mathrm{wt} \%$ of bis(4-dodecylphenyl)iodonium hexafluoroantimonate as an active ingredient. Synthesis of poly(phenyl-co-3-gylcidoxypropylco-perfluorooctyl) silsesquioxane (epoxy-SSQ) was carried out by a previously reported method. ${ }^{[20,21]}$ PTMS, FTES, and GTMS were used to synthesize epoxy-SSQ. The mold release agent $1 \mathrm{H}, 1 \mathrm{H}, 2 \mathrm{H}, 2 \mathrm{H}$ perfluorodecyltrichlorosilane (FDTS) was purchased from Gelest, Inc. (Morrisville, PA, USA). Silquest A-187 silane (GTMS as the main ingredient) was purchased from Crompton Co. (Lisle, IL, USA). PET film with a thickness of $50 \mu \mathrm{m}$ was obtained from $3 \mathrm{M}$ Co. (St Paul, MN, USA). PMMA (average $M_{w} 120$ 000) for encapsulation was purchased from Aldrich.

Instruments: A Nanonex 2000 imprinting tool (Monmouth Junction, NJ, USA) with vacuum capability and UV curing at $365 \mathrm{~nm}$, or a visible light curing system (ELC-430) from Electro-Lite Corporation (Bethel, CT, USA) was used for UV-NIL. aluminium was deposited by electronbeam evaporation. RIE was conducted in a Lam 9400 TCP Poly Etcher tool (Lam Research Corporation, Fremont, CA, USA). Scanning electron microscopy was performed using a Hitachi SU8000 SEM (Tokyo, Japan). Transmission of TM and TE energy was measured using an HR4000CG spectrometer (Ocean Optics, Inc., Dunedin, FL, USA) and a Nikon Eclipse TE300 microscope (Tokyo, Japan) with the assistance of a highquality polarizer. In order to measure the viewing angle, the sample was mounted so it could be tilted to the desired angle. The simulation was performed using the COMSOL program, and by the rigorous coupled wave analysis (RCWA) method. An ellipsometer (AutoEL) was used to measure the refractive index and the thickness of the thin films.

Nanoimprint Process: The formulation of the nanoimprint resist was prepared by dissolving epoxy-SSQ resin in PGMA, followed by the addition of PAG (1 wt\% of the epoxy-SSQ resin). The original silicon oxide master molds were vapor coated with FDTS for easy mold release after the NIL processes. The epoxy-SSQ resin solution was spin coated onto a flexible PET film substrate. The substrates were first surface treated with $\mathrm{O}_{2}$ plasma and then vapor coated with Silquest A-187 silane to promote adhesion to the SSQ resist material. The imprinting process was performed under UV light for a few seconds at room temperature. The Nanonex 2000 imprinting tool or the ELC-430 light curing system was used for imprinting and curing. The imprinting pressure was typically $275 \mathrm{kPa}$.

Aluminium Deposition: The rate of aluminium deposition in this study was $0.5 \mathrm{~nm} / \mathrm{s}$. In order to deposit aluminium on the sidewalls of the imprinted nanopattern but not on the base of the trench, the deposition mount was tilted at $40^{\circ}$. The deposition process was repeated in the opposite direction to deposit aluminium on both sidewalls of the SSQ nanogratings. The thickness of aluminium on both sidewalls was controlled to be $20 \mathrm{~nm}$. This process is illustrated in Figure 1.

Reactive Ion Etching Process: For good polarizer performance, the aluminium layer deposited on the top of the SSQ grating needed to be removed. We used an anisotropic plasma etching process to remove the top layer of aluminium selectively while leaving the aluminium on the sidewalls of the grating relatively intact. aluminium etching was performed using the Lam 9400 tool. The aluminium etching had to be conducted in two steps under $200 \mathrm{~W}$ of transformer-coupled plasma $\mathrm{RF}$ power. First, any residual aluminium oxide layer (thickness, $\sim 1 \mathrm{~nm}$ ) was etched with $40 \mathrm{sccm}$ of $\mathrm{BCl}_{3}$ plasma for $5 \mathrm{~s}$, followed by etching the aluminium layer with $20 \mathrm{sccm} \mathrm{BCl} / 6 \mathrm{sccm} \mathrm{Cl} \mathrm{Cl}_{2}$ plasma.

Encapsulation of WGP: PMMA (0.50 g) was dissolved in $100 \mathrm{~mL}$ of acetone, and the fabricated WGP was dipped in this solution. The fabricated WGP was removed from the PMMA solution and hung vertically for $15 \mathrm{~min}$ at room temperature. This encapsulated WGP was dried in an oven for $60 \mathrm{~min}$ at $60^{\circ} \mathrm{C}$. The coating thickness was controlled to be less than $1 \mu \mathrm{m}$.

\section{Supporting Information}

Supporting Information is available from the Wiley Online Library or from the author. 


\section{Acknowledgements}

The authors would like to thank NSF CMMI and ECCS for the support of this work.

Received: July 3, 2013

Revised: August 20, 2013

Published online: September 17, 2013

[1] J. H. Golden, F. J. DiSalvo, J. Silcox, M. Thomas, J. Elman, Science $1996,273,782$.

[2] X. Liu, X. Deng, J. P. Sciortino, M. Buonanno, F. Walters, R. Varghese, J. Bacon, L. Chen, N. O'Brien, J. J. Wang, Nano Lett. 2006, 6, 2723.

[3] Y. T. Pang, G. W. Meng, L. D. Zhang, Y. Qin, X. Y. Gao, A. W. Zhao, Q. Fang, Adv. Funct. Mater. 2002, 12, 719.

[4] S. W. Ahn, K. D. Lee, J. S. Kim, S. H. Kim, Nanotechnology 2005, 16, 1874.

[5] J. J. Wang, L. Chen, X. M. Liu, P. Sciortino, F. Liu, F. Walters, X. G. Deng, Appl. Phys. Lett. 2006, 89, 141105.

[6] J. J. Wang, F. Walters, X. M. Liu, P. Sciortino, X. G. Deng, Appl. Phys. Lett. 2007, 90, 061104.

[7] A. Pais, A. Banerjee, D. Klotzkin, I. Papautsky, Lab Chip 2008, 8, 794.

[8] J. J. Wang, W. Zhang, X. Deng, J. Deng, F. Liu, P. Sciortino, L. Chen, Optics Lett. 1995, 30, 195.
[9] E. Gardner, D. Hansen, SID Symposium Digest 2003, 62.

[10] Z. Ge, S. T. Wu, Appl. Phys. Lett. 2008, 93, 121104.

[11] M. Suzuki, A. Takada, T. Yamada, T. Hayasaka, K. Sasaki, E. Takahashi, S. Kumagai, Nanotechnology 2010, 21, 175604.

[12] A. E. Hollowell, L. J. Guo, Adv. Opt. Mater. 2013, 1, 343.

[13] Y. Ekinci, H. H. Solak, C. David, H. Sigg, Opt. Express 2006, 14, 2323.

[14] C. C. Hsu, Y. C. Lee, IEEE Int. Nano/Micro Eng. Mol. Systems 2010, 893.

[15] S. Y. Chou, P. R. Krauss, P. J. Renstrom, Appl. Phys. Lett. 1995, 67, 3114.

[16] S. Y. Chou, P. R. Krauss, P. J. Renstrom, Science 1996, 272, 85.

[17] L. J. Guo, Adv. Mater. 2007, 19, 495.

[18] P. Choi, P. F. Fu, L. J. Guo, Adv. Funct. Mater. 2007, 17, 65.

[19] X. Cheng, L. J. Guo, P. F. Fu, Adv. Mat. 2005, 17, 1419.

[20] S. H. Ahn, J. Kim, L. J. Guo, J. Vac. Sci. Technol. B 2007, 25, 2388.

[21] S. H. Ahn, L. J. Guo, Adv. Mater. 2008, 20, 2044.

[22] S. H. Ahn, L. J. Guo, ACS Nano 2009, 3, 2304.

[23] Y. J. Shin, C. Pina-Hernandez, Y. K. Wu, J. G. Ok, L. J. Guo, Nanotechnology 2012, 23, 344018.

[24] J. T. Shen, P. B. Catrysse, S.-H. Fan, Phys. Rev. Lett. 2005, 94, 197401.

[25] C. Pina-Hernandez, P. F. Fu, L. J. Guo, ACS Nano 2011, 5, 923.

[26] C. Pina-Hernandez, L. J. Guo, P. F. Fu, ACS Nano 2010, 4, 4776.

[27] J. Zhou, L. J. Guo, unpublished. 